\title{
Patient safety: are we maximizing the potential of the pharmacist's role? \\ Soraya Dhillon
}

The School of Pharmacy, University of Hertfordshire, Hatfield, UK

In the UK the past 5 years has seen the real emergence of an open, honest culture in healthcare and medical practice which recognizes that healthcare can cause harm to patients. This year has seen the launch of the National Patient Safety campaign which builds on the NHS Adverse Drug Event Collaborative and the Institute of Health Improvement Safer Patients Initiative.[1] The Patient Safety First campaign is being developed in response to the worldwide issue of improving patient safety and is supported by the NHS Institute for Innovation and Improvement, the National Patient Safety Agency (NPSA) and The Health Foundation.[2-4]

The Patient Safety First Campaign for England, by the service, for the service, states its cause as 'To make the safety of patients everyone's highest priority', and its aim as 'No avoidable death and no avoidable harm'. Pharmacists must be linked to this initiative through their organization's participation and currently the campaign is focused on secondary care. The campaign has focused on five key workstreams, [5] as follows. Leadership for safety: getting Boards on board with patient safety, with the aim of demonstrating that it is the highest priority. The goal is to ensure a leadership culture at Board level which promotes quality and patient safety and provides an environment where continuous improvement in harm reduction becomes routine throughout the organization. Care of deteriorating patients in acute care: the goal is to reduce in-hospital cardiac arrest and mortality rates through earlier recognition and treatment of the deteriorating patient.

Critical care bundles (central lines, ventilator care): with the goal of improving the care of patients receiving critical care through the reliable application of care bundles. Perioperative care: including prevention of surgical-site infection and the World Health Organization's Safe Surgery Checklist. The goal is to improve care for adult patients undergoing elective surgical procedures in the hospital setting.

Reduction of harm from high-risk medications: this includes anticoagulants, narcotics, insulin and sedatives. The goal of this workstream is to prevent harm from high-risk medications.

Health professions and services must put patient safety as their number one priority and managers must strive to enable practitioners to keep their patients safe. Pharmacists work within the framework of ensuring that their patients receive safe and effective drug therapy but perhaps only more recently has the service been able to provide this level of practice uniformly. Pharmaceutical departments need to embrace the core principle that patient safety is our number one priority. We must fundamentally question the way in which we organize, manage and deliver our services and question our priorities, as surely it is inappropriate to put our staff in situations of clinical practice where they do not feel safe and cannot ensure the safety of their patients. There are a number of basic factors which need to be examined by pharmaceutical services.

(1) Leadership: do we organize our services to support patient safety? (2) Embedding principles of safe and effective prescribing: have we utilized the breadth of research on effective prescribing, medication errors, medicines reconciliation and discharge planning, and have we implemented these practices? (3) Measurement of clinical interventions: do we use effectively data that we have identified; for example, pharmacy interventions, medicines reconciliation and discharge issues? (4) Implementing change in practice: are all Trusts able to secure appropriate numbers of pharmacists and technicians to ensure that we can implement throughout the organization adequate pharmaceutical support to ensure patient safety? (5) Education and training of pharmacists: undergraduate and postgraduate pharmacy programmes need to demonstrate clear learning outcomes to enhance the pharmacist's understanding of human factors, techniques to minimize adverse drug events and safe and effective prescribing practices.

In the US some of the leading hospitals have harnessed a transformational approach to patient safety. For example, theUS Ascension Health System aims to achieve 'no preventable injuries or deaths in 5 years by end of 2008'.[6] Ascension's approach is a combination of their determination to meet the external regulatory goals, their absolute focus on developing a culture of safety and workstreams which aim to motivate staff to examine and improve their practice. Pharmaceutical services need to embrace this approach.

Pharmacy is a unique discipline with a culture of patient safety, but we have weakness in executing and embedding our role in practice. From drug design to development -that is, in terms of a drug molecule arriving in clinical practice - we have robust regulatory frameworks. We do, however, recognize that we must examine continuously the risks at each stage of the drug-development process in the light of adverse events seen with new biotechnology products at the stage of clinical trials. The availability of and access to medications from abroad requires pharmacists to educate patients to ensure that they understand the regulatory frameworks around products available in Europe and the UK and the potential risks if medicines are purchased in countries where the frameworks may not be as rigorous.

Pharmacists are the key health professionals providing pharmaceutical care services. The evidence base is now strong and we can identify some 500 000-600 000 patients per year admitted to NHS hospitals in England and Wales who may experience a patient safety incident; some untoward events and medication errors are serious enough 
to contribute to unexpected mortality.[7] Medication errors are broadly defined as any error in the prescribing, dispensing or administration of a drug, irrespective of whether such errors lead to adverse consequences or not. They are a growing problem in the UK, although estimates can differ due to the variety of criteria and definitions used: hospital incidence of medication errors may range from 4.4 to 6.2 events per 100 admissions. The NPSA has identified that certain key drug classes - antibiotics, painkillers and sedatives - are likely to be the most common types associated with medication error. The National Patient Safety campaign has identified anticoagulants, narcotics, insulin and sedatives as high-risk medicines, with intravenous drug administration being a particular target.

How strong and robust are our reporting systems? The evidence is clear that we are underestimating the extent of the problem. Medication errors are the single most preventable cause of patient harm. The landmark Institute of Medicine report, To Err is Human: Building a Safer Health System,[8,9] estimated that errors in medical management lead to between 44000 and 98000 deaths in the US each year, although these figures have been questioned. Incident rates of medication errors vary widely, the reason for which can be explained by the different study methods and definitions used. The rate of medication errors varies between 2 and 14\% of patients admitted to hospital, with $1-2 \%$ of patients in the US being harmed as a result, and the majority are due to poor prescribing. Medication error has been estimated to kill 7000 patients per annum and accounts for nearly one in 20 hospital admissions in the US. The incidence is similar in the UK. Medication errors (7\% of all incidents) were the second most common incident reported (after patient falls) in a recent National Audit Office report[10] on patient safety. Pharmacy services need a comprehensive and effective way to identify events that do cause harm to patients. Perhaps we should be fully integrating the Institute of Health Improvement Global Trigger Tool, which is intended to be used to identify all categories of adverse events including those related to medications.[11] The history of the development of clinical pharmacy, pharmaceutical care and medicines management put the pharmacist in a unique position to play a lead role in patient safety by ensuring medication safety for patients. Fundamental to our role is ensuring we have rational and effective guidelines and formulary management systems. What is sad is that work in the late 1980s and early 1990s recognized the importance of formulary management of antibiotic prescribing, yet it is only over the past few years that organizations have embedded antibiotic prescribing policies to manage the risks of MRSA and Clostridium difficile.

Pharmacists need to ensure that patients can manage their medicines effectively though their role in the drug-use process. Practice research has now provided an evidence base which shows how electronic prescribing. medicines reconciliation and pharmaceutical care in discharge planning can ensure the effectiveness and safety of medicines use. However, in 2008 we are only now starting to develop the IT infrastructure for electronic prescribing and discharge, we have not embedded effective pharmaceutical care in discharge-management processes, and still patients do not take their medicines appropriately and have significant medication-related problems. Pharmacy developments must now implement more effectively our service principles and goals of delivering pharmaceutical care to ensure that patients are safe and to achieve effective health outcomes.

Pharmacists must not take 20 years to embrace patient safety tools. Managers must play a crucial leadership role in creating a culture of patient safety which transforms the delivery of effective pharmaceutical services, putting patient safety as the number one priority.

\section{References}

1. National Patient Safety Agency (2008). www.npsa.nhs.uk/ (accessed 29 November 2008).

2. Patient Safety First Campaign (2008). www.patientsafetyfirst. nhs.uk/Content.aspx?path=/ (accessed 29 November 2008).

3. Institute of Innovation and Improvement (2008). Safer Care.

Improving patient safety. www.institute.nhs.uk/safer_care/safer_ care/safer_care_-_home_page_2.html (accessed 29 November 2008).

4. The Health Foundation (2008). www.health.org.uk/ (accessed 29 November 2008).

5. Patient Safety First Campaign (2008). www.patientsafetyfirst. nhs.uk/Content.aspx? path=/ (accessed 29 November 2008).

6. Us Ascension Health system. http://www.ascensionhealth.org/ ht_safe/main.asp (accessed 8th December 2008).

7. National Patient Safety Agency. Building a Memory: Preventing Harm, Reducing Risk and Improving Patient Safety. The

First Report of the National Reporting and Learning System and the Patient Safety Observatory. London: NPSA, 2005.

8. Institute of Medicine (IOM) report USA (2000). http://www.nap. edu/openbook.php?isbn=0309068371 (accessed 8th December 2008).

9. Kohn LT, Corrigaan JM, Donalsdon MS, eds. To Err Is Human: 
Building a Safer Health System. Committee on Quality of Health Care in America, Institute of Medicine (IOM). http://www.nap. edu/catalog.php?record_id=9728 (accessed 8th December 2008). 10. National Audit Office. A Safer Place for Patients: Learning to Improve Patient Safety http://www.nao.org.uk/publications/ nao_reports/05-06/0506456.pdf (accessed 8th December 2008).

11. Adverse Drug Event Trigger Tool: A Practical Methodology for Measuring Medication Related Harm. http://www.ihi.org/IHI/

Topics/PatientSafety/SafetyGeneral/Tools/IHIGlobalTrigger-

ToolforMeasuringAEs.htm (accessed 8th December 2008). 